117:1616-1626. doi:10.1172/JCI31581.

4. Eghbali-Fatourechi, G.Z., et al. 2005. Circulating osteoblast-lineage cells in humans. N. Engl. J. Med. 352:1959-1966.

5. Bouletreau, P.J., et al. 2002. Hypoxia and VEGF up-regulate BMP-2 mRNA and protein expression in microvascular endothelial cells: implications for fracture healing. Plast. Reconstr. Surg. 109:2384-2397.

6. Gerstenfeld, L.C., et al. 2003. Impaired fracture healing in the absence of TNF-alpha signaling: the role of TNF-alpha in endochondral cartilage resorption. J. Bone Miner. Res. 18:1584-1592.

7. Sorescu, G.P., et al. 2004. Bone morphogenic protein 4 produced in endothelial cells by oscillatory shear stress induces monocyte adhesion by stimulating reactive oxygen species production from a nox1-based NADPH oxidase. Circ. Res. 95:773-779.

8. Csiszar, A., et al. 2006. Bone morphogenetic protein-2 induces proinflammatory endothelial phenotype. Am. J. Pathol. 168:629-638.

9. Ferrara, N. 2004. Vascular endothelial growth factor: basic science and clinical progress. Endocr. Rev. 25:581-611.

10. Zelzer, E., and Olsen, B.R. 2005. Multiple roles of vascular endothelial growth factor (VEGF) in skeletal development, growth, and repair. Curr. Top. Dev. Biol. 65:169-187.

11.Zelzer, E., et al. 2002. Skeletal defects in VEGF(120/120) mice reveal multiple roles for VEGF in skeletogenesis. Development. 129:1893-1904.

12. He, Y., et al. 2006. Critical function of Bmx/Etk in ischemia-mediated arteriogenesis and angiogenesis. J. Clin. Invest. 116:2344-2355. doi:10.1172/ JCI28123.

13. Goukassian, D.A., et al. 2007. Tumor necrosis factor- alpha receptor $\mathrm{p} 75$ is required in ischemia-induced neovascularization. Circulation. 115:752-762.

14. Ratcliffe, P.J. 2007. HIF-1 and HIF-2: working alone or together in hypoxia? J. Clin. Invest. 117:862-865. doi:10.1172/JCI31750.

15. Forsythe, J.A., et al. 1996. Activation of vascular endothelial growth factor gene transcription by hypoxia-inducible factor $1 . \mathrm{Mol}$. Cell. Biol. 16:4604-4613

16. Shi, S., and Gronthos, S. 2003. Perivascular niche of postnatal mesenchymal stem cells in human bone marrow and dental pulp. J. Bone Miner. Res. 18:696-704.

17. Farrington-Rock, C., et al. 2004. Chondrogenic and adipogenic potential of microvascular pericytes. Circulation. 110:2226-2232.

18. Tintut, Y., et al. 2003. Multilineage potential of cells from the artery wall. Circulation. 108:2505-2510.

19. Schor, A.M., Allen, T.D., Canfield, A.E., Sloan, P., and Schor, S.L. 1990. Pericytes derived from the retinal microvasculature undergo calcification in vitro. J. Cell Sci. 97:449-461.

20. Brunelli, S., et al. 2004. Msx2 and necdin combined activities are required for smooth muscle differentiation in mesoangioblast stem cells. Circ. Res. 94:1571-1578

21. Shao, J.S., et al. 2005. Msx2 promotes cardiovascular calcification by activating paracrine Wn signals. J. Clin. Invest. 115:1210-1220. doi:10.1172/ JCI200524140.

22. Cheng, S.L., Shao, J.S., Charlton-Kachigian, N Loewy, A.P., and Towler, D.A. 2003. MSX2 promotes osteogenesis and suppresses adipogenic differentiation of multipotent mesenchymal progenitors. J. Biol. Chem. 278:45969-45977.

23. Sakurai, H., et al. 2006. In vitro modeling of par- axial and lateral mesoderm differentiation reveals early reversibility. Stem Cells. 24:575-586.

24. Kalajzic, I., et al. 2006. Myofibroblast/pericyte phenotype of the osteoprogenitor cell. J. Bone Miner. Res. 21:S2, abstract 1004.

25. Eames, B.F., de la Fuente, L., and Helms, J.A. 2003. Molecular ontogeny of the skeleton. Birth Defects Res. C Embryo Today. 69:93-101.

26. Burrows, A.M., et al. 2001. Endocranial vascular patterns in a familial rabbit model of coronal suture synostosis. Cleft Palate Craniofac. J. 38:615-621.

27. Henderson, J.H., Longaker, M.T., and Carter, D.R. 2004. Sutural bone deposition rate and strain magnitude during cranial development. Bone. 34:271-280.

28. Fong, K.D., et al. 2003. Mechanical strain affects dura mater biological processes: implications for mmature calvarial healing. Plast. Reconstr. Surg. 112:1312-1327

29. Carvalho, R.S., et al. 2004. The role of angiogenesis in a murine tibial model of distraction osteogenesis. Bone. 34:849-861.

30. George, D.J., and Kaelin, W.G., Jr. 2003. The von Hippel-Lindau protein, vascular endothelial growth factor, and kidney cancer. N. Engl. J. Med. 349:419-421.

31. Esner, M., et al. 2006. Smooth muscle of the dorsal aorta shares a common clonal origin with skeletal muscle of the myotome. Development. 133:737-749

32. Tagliafico, E., et al. 2004. TGFbeta/BMP activate the smooth muscle/bone differentiation programs in mesoangioblasts. J. Cell Sci. 117:4377-4388.

33. Cossu, G., and Bianco, P. 2003. Mesoangioblasts - vascular progenitors for extravascular mesodermal tissues. Curr. Opin. Genet. Dev. 13:537-542.

\title{
Sizing up sialic acid in glomerular disease
}

\author{
Susan E. Quaggin \\ The Samuel Lunenfeld Research Institute, Mount Sinai Hospital, and Division of Nephrology, St. Michael's Hospital, \\ University of Toronto, Toronto, Ontario, Canada.
}

\begin{abstract}
A new study by Galeano and colleagues in this issue of the JCI reports the first glomerular disease caused by a genetic defect in sialic acid biosynthesis (see the related article beginning on page 1585). Mice that harbor mutations in the Gne/Mnk gene produce lower amounts of sialic acid, suffer from hematuria, proteinuria, and structural defects in the glomerulus and die within days after birth. Remarkably, the lesion can be reversed through dietary addition of $N$-acetylmannosamine, a sialic acid precursor, raising the intriguing possibility that this approach might have therapeutic benefit in patients with glomerular disease.
\end{abstract}

In this issue of the JCI, Galeano, Huizing, and colleagues (1) describe kidney defects in knockin mice that harbor the M712T muta-

Nonstandard abbreviations used: Gne, uridine diphospho- $\mathrm{N}$-acetylglucosamine 2-epimerase; HIBM, hereditary inclusion body myopathy; ManNAc, $N$-acetylmannosamine; Mnk, ManNAc kinase; PC, podocalyxin; SD, slit diaphragm.

Conflict of interest: The author is the recipient of a research grant from Genzyme and is a scientific consultant for Genentech.

Citation for this article: J. Clin. Invest. 117:1480-1483 (2007). doi:10.1172/JCI32482. tion in the gene encoding the key bifunctional enzyme of sialic acid biosynthesis - uridine diphospho- $N$-acetylglucosamine 2-epimerase/ $\mathrm{N}$-acetylmannosamine (ManNAc) kinase (GNE/MNK) (Gne $e^{M 712 T / M 712 T}$ mice) $(2,3)$. In patients, mutations in the GNE gene result in the autosomal recessive neuromuscular disorder, hereditary inclusion body myopathy (HIBM; MIM 600737) that presents late in life as a slowly progressive myopathy (4, 5). The $N$-acetylglucosamine/ ManNAc enzyme is ubiquitously expressed and catalyzes the first rate-limiting steps in the biosynthesis of sialic acid (Figure 1). $5-\mathrm{N}$-acetylneuraminic acid (Neu5Ac) is the most plentiful mammalian sialic acid and is the terminal sugar on glycoconjugates, where it functions in cellular interactions and signaling. Muscle fibers from patients with HIBM exhibit reduced sialylation of proteins, which is believed to underlie disease pathogenesis. Currently, there is no effective therapy for this disorder.

In the current study (1), the investigators sought to develop a mouse model to test whether dietary supplementation of sialic acid or its precursor, ManNAc, could reverse the hyposialylation defect observed in patients. Standard Gne knockout mice die in utero (6). To overcome this early mortality, the authors generated a mouse carrying one of the most common nonlethal mutations observed in patients with HIBM. Surprisingly, mice homozygous for 
Cell membrane

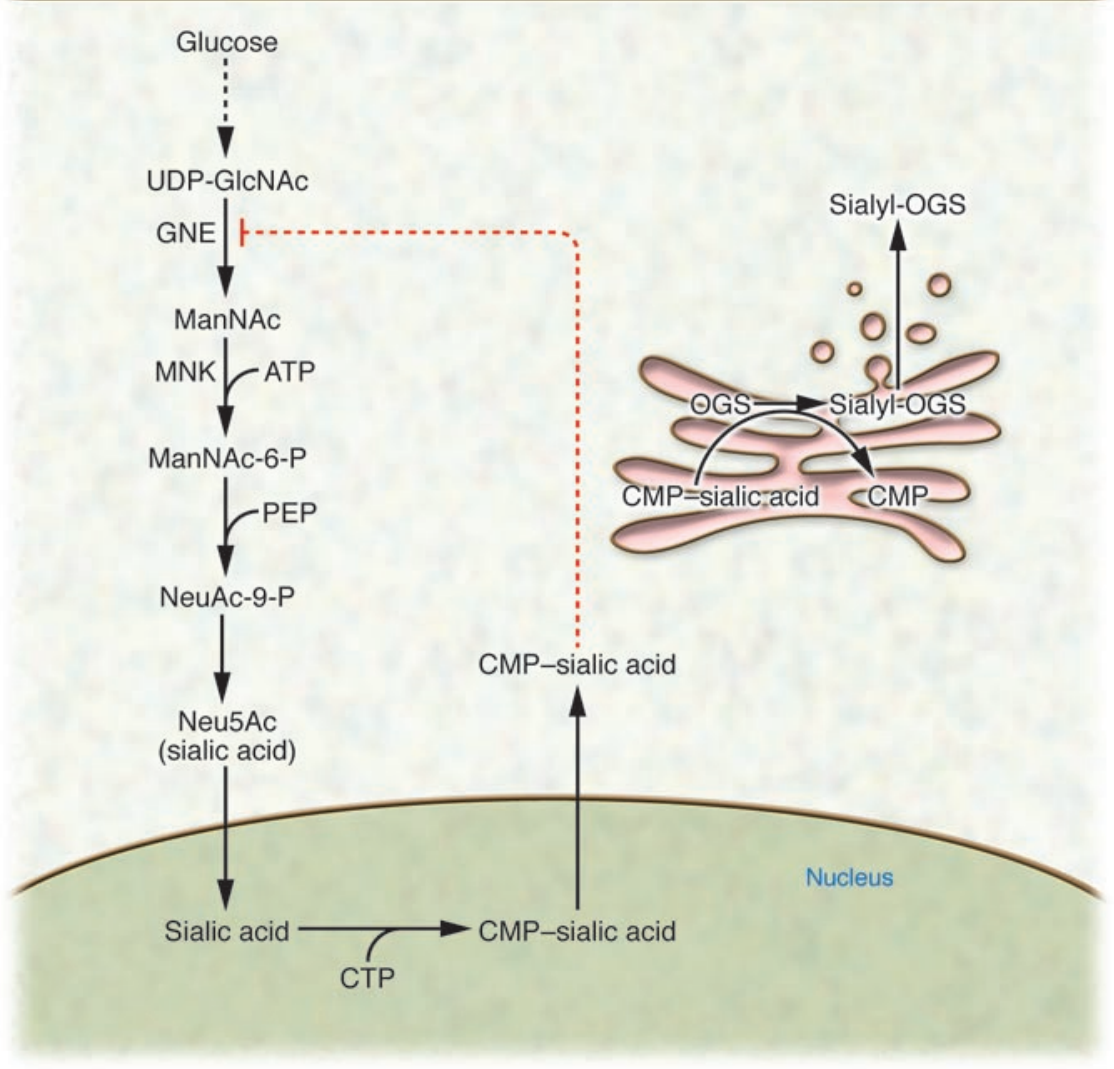

the $G n e^{M 712 T}$ allele died within days of birth with major defects in the glomerulus of the kidney. They did not survive long enough to determine whether they would develop any muscle disease late in life. Patients with HIBM are not reported to have glomerular disease or abnormalities in renal function. Given the discrepancy in phenotype between species, the most obvious first question is whether sialylation defects in the kidney can explain the glomerular defect.

\section{Sialylation defects and models of glomerular disease}

The glomerular filtration barrier separates the blood from the urinary space and is comprised of renal podocytes (glomerular visceral epithelial cells), fenestrated endothelial cells, and an intervening glomerular basement membrane $(7,8)$ (Figure 2). Each kidney contains roughly 1 million individual glomeruli that together produce 180 liters of filtrate per day. The barrier restricts passage of large macromolecules such as albumin and cells while permitting free passage to water and small solutes (9). The most abundant and best-studied sialoglycoprotein in the glomerulus is podocalyxin (PC) (10). PC is found on glomerular endothelial cells and on the apical or freefloating surface of podocytes, where it connects to the actin cytoskeleton through $\mathrm{Na}^{+} / \mathrm{H}^{+}$exchanger regulatory factor 2 (NHERF2) and phosphorylated ezrin (11, 12). Loss of the interaction between PC and the actin cytoskeleton is associated with the nephrotic syndrome, a disorder characterized by proteinuria and dramatic structural change of the podocytes due to collapse and flattening of the actin-based foot processes (13). Loss of sialylation of PC occurs in rodents injected with sialidase, puromycin aminonucleoside, or protamine sulfate, which neutralizes negative charges (14-16). All of these compounds cause an abrupt onset of proteinuria and foot process fusion. Simultaneous infusion of sialic acid prevents the proteinuria and podocyte effacement observed with puromycin injection, presumably due to resialylation of critical glomerular proteins (17). Taken together, these findings underscore the importance of protein sialylation in the control of glomerular structure and function.

\section{Genetic model for hyposialylation}

Mice homozygous for the Gne $e^{M 712 T / M 712 T}$ mutation die within days of birth with

\section{Figure 1}

The biochemical pathway of sialic acid formation. CMP, cytidine monophosphate; MaNAc-6-P, MaNAc-6-phosphate; NeuAc-9-P, N-acetylneuraminic acid-9-phosphate; sialyl-OGS, sialylated oligosaccharides; PEP, phosphoenolpyruvate; UDP-GIcNAC, uridine diphospho$N$-acetylglucosamine. Adapted from Galeano et al. (1). CTP, cytidine triphosphate.

growth retardation and major structural abnormalities in the kidneys. The urinary space and renal tubules in these mice are packed with red blood cells and protein without any obvious inflammation, suggesting a major defect in formation and function of the glomerular filtration barrier. Accordingly, electron micrographs show segmental splitting of the glomerular basement membrane and fusion or absence of foot processes (Figure 2). Formation of the foot processes requires slit diaphragm (SD) proteins that are found in specialized intercellular junctions between foot processes. Phosphorylation of the SD protein nephrin (NPHS1) results in recruitment of Nck (noncatalytic region of tyrosine kinase) $\mathrm{SH}_{2}$-containing adaptor proteins and actin cytoskeletal reorganization (18-21). Although Galeano et al. (1) show that the SD proteins NPHS1 and podocin (NPHS2) are expressed in glomeruli from Gne mutants at wild-type levels, their subcellular localization and/or phosphorylation status were not examined.

Sialylation of PC is reduced in glomeruli from Gne $e^{M 712 T / M 712 T}$ mutant mice (1). Because PC is known to associate with actin cytoskeletal proteins in the podocytes, and loss of this interaction disrupts glomerular barrier function, hyposialylation might explain the renal defect in the Gne $e^{M 712 T / M 712 T}$ mutants. However, there are significant differences in phenotypes between PC KO mice and the Gne $e^{M 712 T / M 712 T}$ mutants. PC $\mathrm{KO}$ mice also die in the perinatal period with renal defects that include failure of SD formation, fusion of foot processes, and a complete lack of filtration with no urine in the bladder (21). However, no splitting of the glomerular basement membrane or leakage of red blood cells into the renal tubules was observed. In addition, PC $\mathrm{KO}$ mice exhibit extrarenal defects such as hernias and omphaloceles (protrusions of the intestine and omentum through a hernia in the abdominal wall near the navel) due to the broad expression of PC during fetal life. By contrast, no other defects were observed in the Gne $e^{M 712 T / M 712 T}$ mutants in any other organs including muscle, heart, 

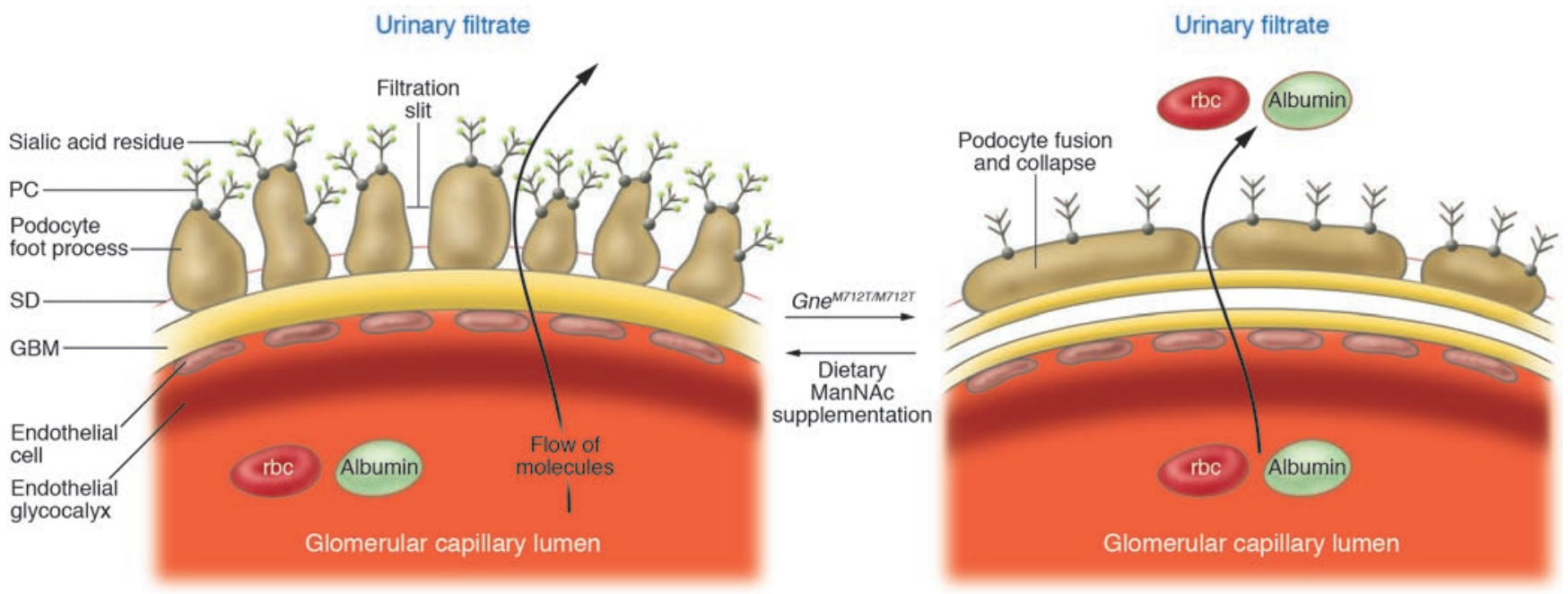

Figure 2

The glomerular filtration barrier, with and without sialylated proteins. Blood enters the glomerular capillaries and is filtered across the endothelium and the basement membrane and through the filtration slits between podocyte foot processes to produce the primary urinary filtrate. In healthy glomeruli, this barrier restricts the passage of macromolecules but is highly permeable to water and small solutes. In this issue of the $\mathrm{JCl}$, Galeano et al. (1) show that a mutation (M712T) in Gne/Mnk in mice results in a reduction in the number of sialic acid residues on critical glomerular proteins such as PC that are found on the apical surface of podocytes. Loss of sialylated proteins is associated with foot process fusion or collapse, splitting of the glomerular basement membrane (GBM), and loss of rbc and proteins such as albumin into the urine. Dietary supplementation with ManNAc prolongs life in mutant Gne ${ }^{M 712 T / M 712 T}$ mice and improves ultrastructure of the glomerular filtration barrier.

or lungs. Furthermore, Gne KO mice rescued by a Gne transgene harboring another common mutation (V572L, which is found in patients of Japanese origin with HIBM) survived but develop muscle defects and fibrosis of the diaphragm and heart. Interestingly, some of these mice died suddenly, although the cause was unclear. Although the authors stated that other internal organs had no defects, analysis of renal function was not described in detail (22).

Why are the glomerular defects apparently more profound in Gne $e^{M 712 T / M 712 T}$ mutants than in PC KO mice or other animal models of sialylation defects? Several possible explanations exist. While PC is expressed by the podocyte, the endothelium and glomerular basement membranes also make significant contributions to the glomerular filtration barrier, and it is reasonable to assume that proteins in these compartments may also require sialic acid for normal functioning. In keeping with this hypothesis, PC is also expressed by endothelial cells, and splitting of the glomerular basement membrane (with hematuria) seen in the Gne $e^{M 712 T / M 712 T}$ mutants is reminiscent of the lesion seen in Alport syndrome, a disease caused by mutations in the collagen type IV $\alpha 5$ (COL4a5), COL4a4, and $C O L 4 a 3$ genes; the proteins encoded by these genes are major constituents of the glomerular basement membrane (23).

\section{Toward a novel treatment for glomerular diseases}

Strikingly, Galeano et al. (1) show that supplementation of the pregnant mouse's diet with ManNAc, a precursor to sialic acid that readily crosses cell membranes, prolongs survival of the Gne $e^{M 712 T / M 712 T}$ mice. This treatment was associated with an almost 10 -fold enhancement of GNE enzyme activity in the kidney. Furthermore, sialylation of PC was also significantly increased in the kidneys of treated mutants, and there was marked improvement in ultrastructure of the filtration barrier.

Admittedly, there are striking differences between the human and mouse phenotypes caused by the identical GNE/Gne mutant alleles. Further understanding of the nature of this species difference could provide important insights into the regulation of protein sialylation in the kidney and its contribution to filtration barrier function. There is abundant evidence that human glomerular proteins are sialylated and that alterations in sialylation patterns can be associated with disease, although sialylation defects of individual proteins have not been reported. Thus, based on the dramatic effects of dietary ManNAc supplementation in this very aggressive model of glomerular pathology along with the simplicity and tolerability of this intervention, it seems plausible that this pathway might be useful as a more general therapeutic approach for glomerular diseases of many causes. This approach is especially attractive, as current therapies are nonspecific, associated with significant adverse side effects, and are often ineffective.

\section{Acknowledgments}

S.E. Quaggin thanks Dontscho Kerjaschki for critical review and helpful comments. S.E. Quaggin is the recipient of a Canada Research Chair Tier II, an Emerald Foundation award, and a Premier's of Ontario Research of Excellence Award. This work was funded through Canadian Institutes of Health Research grant MOP 77756, National Cancer Institute of Canada grant 16002, and a Kidney Foundation of Canada Award.

Address correspondence to: Susan E. Quaggin, The Samuel Lunenfeld Research Institute, Room 855Q, Mount Sinai Hospital, 600 University Avenue, Toronto, Ontario M5G 1X5, Canada. Phone: (416) 586-4800 ext. 2859; Fax: (416) 586-8588; E-mail: quaggin@mshri.on.ca.

1. Galeano, B., et al. 2007. Mutation in the key enzyme of sialic acid biosynthesis causes severe glomerular proteinuria and is rescued by $N$-acetlymannosamine. J. Clin. Invest. 117:1585-1594. doi:10.1172/JCI30954.

2. Hinderlich, S., et al. 1997. A bifunctional enzyme catalyzes the first two steps in $\mathrm{N}$-acetylneuraminic acid biosynthesis of rat liver. Purification and char- 
acterization of UDP-N-acetylglucosamine 2-epimerase/ $\mathrm{N}$-acetylmannosamine kinase. J. Biol. Chem. 272:24313-24318.

3. Keppler, O.T., et al. 1999. UDP-GlcNAc 2-epimerase: a regulator of cell surface sialylation. Science. 284:1372-1376.

4. Eisenberg, I., et al. 2001. The UDP-N-acetylglucosamine 2-epimerase/ $\mathrm{N}$-acetylmannosamine kinase gene is mutated in recessive hereditary inclusion body myopathy. Nat. Genet. 29:83-87.

5. Eisenberg, I., et al. 2003. Mutations spectrum of GNE in hereditary inclusion body myopathy sparing the quadriceps. Hum. Mutat. 21:99.

6. Schwarzkopf, M., et al. 2002. Sialylation is essential for early development in mice. Proc. Natl. Acad. Sci. U. S. A. 99: 5267-5270.

7. Tryggvason, K. 1999. Unraveling the mechanisms of glomerular ultrafiltration: nephrin, a key component of the slit diaphragm. J. Am. Soc. Nephrol. 10:2440-2445.

8. Somlo, S., and Mundel, P. 2000. Getting a foothold in nephrotic syndrome. Nat. Genet. 24:333-335.

9. Kriz, W., et al. 1996. Stability and leakiness: opposing challenges to the glomerulus. Kidney Int. 49:1570-1574.

10. Kerjaschki, D., Sharkey, D.J., and Farquhar, M.G. 1984. Identification and characterization of podo- calyxin - the major sialoprotein of the renal glomerular epithelial cell. J. Cell Biol. 98:1591-1596.

11. Schmieder, S., Nagai, M., Orlando, R.A., Takeda, T., and Farquhar, M.G. 2004. Podocalyxin activates RhoA and induces actin reorganization through NHERF1 and Ezrin in MDCK cells. J. Am. Soc. Nephrol. 15:289-298.

12. Orlando, R.A., et al. 2001. The glomerular epithelial cell anti-adhesin podocalyxin associates with the actin cytoskeleton through interactions with ezrin. J. Am. Soc. Nephrol. 12:1589-1598.

13. Takeda, T., et al. 2001. Loss of glomerular foot processes is associated with uncoupling of podocalyxin from the actin cytoskeleton. J. Clin. Invest. 108:289-301. doi:10.1172/JCI200112539.

14. Seiler, M.W., et al. 1977. Pathogenesis of polycation-induced alterations ("fusion") of glomerular epithelium. Lab. Invest. 36:48-61.

15. Kerjaschki, D., Vernillo, A.T., and Farquhar, M.G. 1985. Reduced sialylation of podocalyxin - the major sialoprotein of the rat kidney glomerulus - in aminonucleoside nephrosis. Am. J. Pathol. 118:343-349.

16. Kurihara, H., Anderson, J.M., Kerjaschki, G., and Farquhar, M.G. 1992. The altered glomerular filtration slits seen in puromycin aminonucleoside nephrosis and protamine sulfate-treated rats con- tain the tight junction protein ZO-1. Am. J. Pathol. 141:805-816.

17. Muchitsch, E., et al. 1999. Effects of human alpha-1acid glycoprotein on aminonucleoside-induced minimal change nephrosis in rats. Nephron. 81:194-199.

18. Kestila, M., et al. 1998. Positionally cloned gene for a novel glomerular protein - nephrin - is mutated in congenital nephrotic syndrome. Mol. Cell. 1:575-582.

19. Verma, R., et al. 2006. Nephrin ectodomain engagement results in Src kinase activation, nephrin phosphorylation, Nck recruitment, and actin polymerization. J. Clin. Invest. 116:1346-1359. doi:10.1172/JCI27414.

20. Jones, N., et al. 2006. Nck adaptor proteins link nephrin to the actin cytoskeleton of kidney podocytes. Nature. 440:818-823.

21. Doyonnas, R., et al. 2001. Anuria, omphalocele, and perinatal lethality in mice lacking the CD34-related protein podocalyxin. J. Exp. Med. 194:13-27.

22. Malicdan, M.C., et al. 2007. A Gne knockout mouse expressing human V572L mutation develops features similar to distal myopathy with rimmed vacuoles or hereditary inclusion body myopathy. Hum. Mol. Genet. 16:115-128.

23. Barker, D.F., et al. 1990. Identification of mutations in the COL4A5 collagen gene in Alport syndrome. Science. 248:1224-1227.

\section{A beneficial role for IL-1 $\beta$ in Alzheimer disease?}

\section{Cynthia A. Lemere}

Center for Neurologic Diseases, Brigham and Women's Hospital, Harvard Medical School, Boston, Massachusetts, USA.

\begin{abstract}
While the term neuroinflammation often conjures up images of cellular damage, mounting evidence suggests that certain proinflammatory molecules, such as the cytokine IL-1 $\beta$, may have beneficial and protective effects. In a report in this issue of the JCI, Shaftel and coworkers have generated an elegant mouse model in which local hippocampal overexpression of IL-1 $\beta$ in an Alzheimer disease (AD) transgenic mouse model resulted not in the expected exacerbation of the amyloid $\beta$ plaque deposition common to $A D$, but instead in plaque amelioration (see the related article beginning on page 1595). Thus, manipulation of the immune system may be a potential therapeutic approach to protect against $\mathrm{AD}$, although further studies are needed to understand all of the downstream effects of this manipulation.
\end{abstract}

The role of neuroinflammation in Alzheimer disease $(\mathrm{AD})$ remains somewhat of a mystery. Although inflammatory proteins such as cytokines, chemokines, complement, and acute phase proteins are elevated in $\mathrm{AD}$

Nonstandard abbreviations used: $A \beta$, amyloid $\beta$; $\mathrm{AD}$, Alzheimer disease; APP, amyloid precursor protein; APP/PS1+IL- $1 \beta$ mouse, mouse expressing the $\mathrm{APP} / \mathrm{PS} 1$ and IL-1 $\beta^{\mathrm{XAT}}$ transgenes; FIV, feline immunodeficiency virus; Iba-1, ionized calcium-binding adaptor molecule 1; PS1, presenilin 1; XAT, excisional activation transgene.

Conflict of interest: The author received partial research funding from Elan Corporation and Wyeth Pharmaceuticals to study $A \beta$ immunotherapy during the past 3 years. The author's spouse is an employee and stockholder of Genzyme.

Citation for this article: J. Clin. Invest. 117:1483-1485 (2007). doi:10.1172/JCI32356. brain, the status of each as a "good guy" or a "bad guy" is still unclear (1). On one hand, inflammation may be a secondary response to progressive neurodegeneration in the brain. In this instance, immune cells in the brain, such as microglia and astrocytes, may become activated and secrete inflammatory molecules that may then further accelerate pathogenesis (2). On the other hand, recent evidence points to a potentially beneficial and protective role of inflammation in the brain (3). In this case, inflammation appears to induce microglial phagocytosis of amyloid $\beta(A \beta)$ protein, reducing its deposition into plaques. $A \beta$ protein is proteolytically cleaved from its large precursor, amyloid precursor protein (APP), and secreted; in $\mathrm{AD}, \mathrm{A} \beta$ accumulates extracellularly into plaques, a hallmark pathological feature of the disease (4).

In a study reported in this issue of the JCI, Shaftel and coworkers present provocative new findings in support of the latter scenario (5). The authors overexpressed the proinflammatory cytokine IL- $1 \beta$ (IL-1 $\beta$ ) in a region- and time-dependent fashion in transgenic mice overexpressing human mutant APP and presenilin 1 (APP/PS1 mice), a mouse model designed to partially mimic AD pathology in which extracellular $A \beta$ plaques accumulate in the brain with aging. Excisional activation transgene (XAT) technology was used to first generate IL-1 $\beta^{\mathrm{XAT}}$ transgenic mice (5). Upon injection of feline immunodeficiency virus-Cre (FIV-Cre) to locally activate the IL-1 $\beta$ transgene in brain, these mice were shown to have elevated levels of IL-1 $\beta$ and glial fibrillary acid protein (GFAP), indicative of astrocytic activation, as well as of ionized calcium-binding adaptor molecule 1 (Iba-1) and MHC class II, indicative of microglial activation. Expression of the transgene was still apparent 1 year after injection. These mice were then crossbred with APP/PS1 mice, and the resulting mice expressing the APP/PS1 and IL- $1 \beta^{\text {XAT }}$ transgenes (APP/PS $1+$ IL- $\beta$ 